\title{
OBSERVATION OF SLEEP-RELATED BREATHING DISORDERS IN PATIENTS WITH CORONARY ARTERY DISEASE BY AMBULATORY ELECTROCARDIOGRAM-RESPIRATION MONITORING SYSTEM
}

\author{
Osamu Tateishi, M.D., Tetsuo Okamura, M.D., Tetsushi Itou, M.D. \\ Michio Murakami, M.D., Takeshi Suda, M.D., Izumi Nishimuta, M.D. \\ Shin-ichirou Obata, M.D. and Takayuki Nagata, M.D.
}

\begin{abstract}
Eighty-five coronary artery patients examined using an ambulatory electrocardiogram-respiration monitoring system (AERMS) in which a respiratory sensor was strapped to the right upper abdominal wall. Apnea was defined as a cessation of abdominal wall movement lasting at least $10 \mathrm{sec}$. Sleep-related breathing disorder (SRBD) was diagnosed if at least 30 apneic episodes were observed during sleep. The cardiac events evaluated during follow-up included occurrence of sudden death, myocardial infarction and ventricular tachycardia.

SRBD was detected in 9 of 85 patients $(11 \%)$. There were more patients with low $\mathrm{EF}(\mathrm{EF}<50 \%)$ in the SRBD group than in the non-SRBD group $(\mathrm{p}<0.01)$. During follow-up for a mean period of $18.4 \pm 7.6$ months after ambulatory recording, four of nine (44\%) patients in the SRBD group had cardiac events, compared with only four of $79(6 \%)$ patients in the non-SRBD group $(\mathrm{p}<0.001)$.

Thus, coronary artery patients who were complicated with SRBD showed poor cardiac function and had a high incidence of cardiac events.
\end{abstract}

(Jpn Circ J 1994; 58: 831-835)

$\mathbf{R}^{\mathrm{r}}$ ECENT advances in the study of sleeprelated breathing disorder (SRBD) have revealed that hemodynamic disturbances such as hypoxia, elevation of blood pressure and changes in heart rate occur and various arrhythmias appear during sleep in patients with sleep apnea syndrome (SAS) $!^{-4}$ Therefore, the occurrence of sleep apnea in patients with coronary artery disease (CAD) could worsen the prognosis. However, the diagnosis of SRBD by polysomnography requires a great deal of time and effort for both the physician and patient.

Key words:

Sudden cardiac death

Arrhythmia

Morbidity
Recently, Otsuka et alș reported an ambulatory electrocardiogram-respiration monitoring system (AERMS) for conveniently detecting SRBD. To examine SRBD in a more convenient manner, we used AERMS on an outpatient basis to diagnose sleep apnea and further investigated the clinical characteristics of SRBD patients with coronary artery diseases.

\section{METHODS}

\section{Subjects}

Eighty-five patients underwent ambulatory electrocardiogram-respiration recordings. These patients consisted of 81 men and 4

(Received October 16, 1993; accepted March 28, 1994)

Department of Internal Medicine (IV), The Jikei University School of Medicine

Mailing address: Osamu Tateishi, M.D., Department of Internal Medicine (IV), The Jikei University School of Medicine, 3-25-8 Nishishinbashi Minato-ku Tokyo 105, Japan 


\section{4-hour Holter recording}

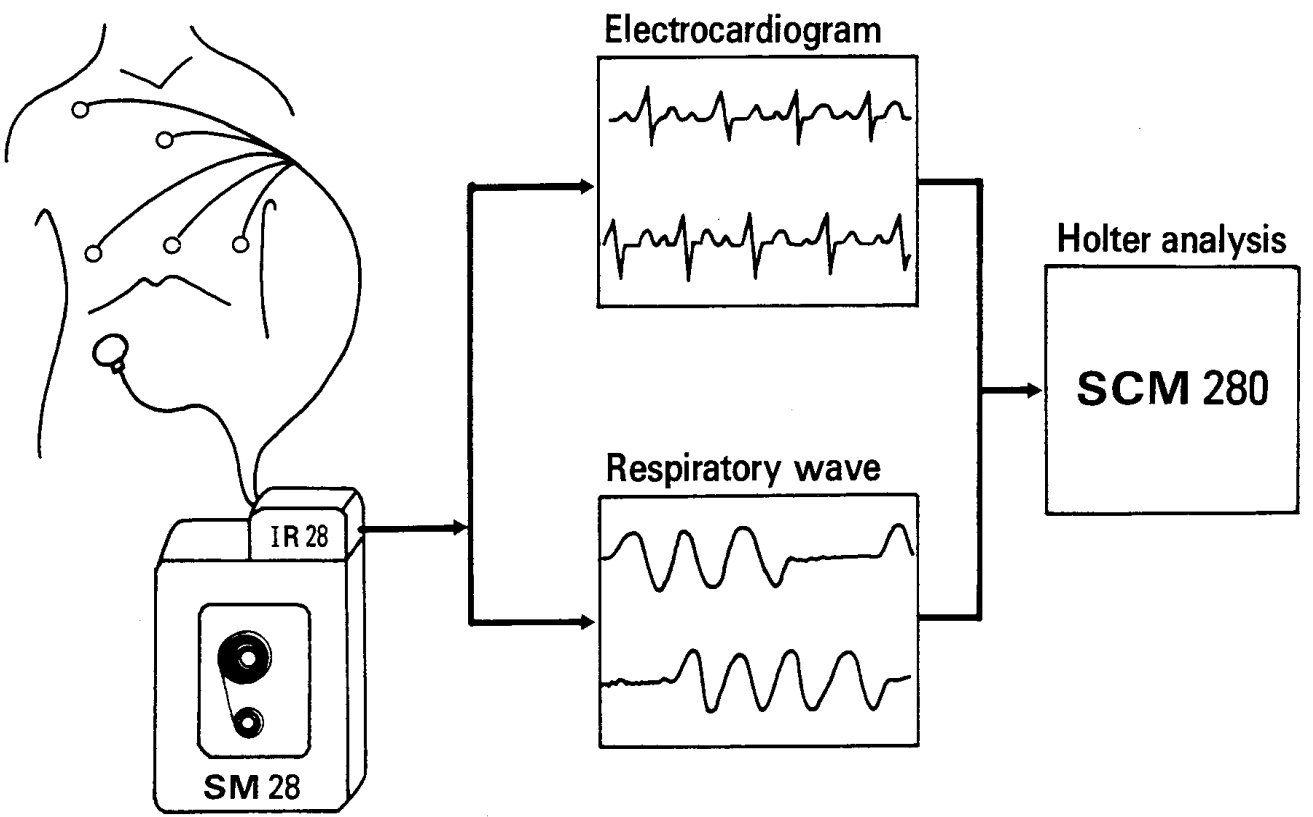

Fig.1. Ambulatory electrocardiogram-respiration monitoring system.

A respiratory wave was recorded using a respiratory monitoring apparatus.

The air bag sensor was strapped to the right upper abdominal wall.

women, with a mean age of $57 \pm 10$ years. Thirty-eight had old myocardial infarction and 47 had chronic stable angina pectoris. Criteria for diagnosis were typical evolution of ST.T abnormalities and a rise in pertinent enzymes for myocardial infarction, and chest pain evoked by exercise and positive ischemic change in stress exercise electrocardiography or scintigraphy for angina pectoris. All of the patients underwent diagnostic coronary angiography and contrast cineangiography. Coronary artery stenosis was considered as significant with stenosis of the lumen of more than $75 \%$. The ejection fraction (EF) was considered low at $\mathrm{EF}<50 \%$. Informed consent was obtained from all of the patients before study.

\section{Holter Monitoring}

Twenty-four-hour ambulatory tape recordings were obtained using a commercially available three-channel recorder (Fukuda SM28). Two electrocardiograms (NASA and CC5 lead) were recorded on the first and second channels and a respiratory wave was simultaneously recorded on the third channel. The respiratory wave was recorded using a respiration monitoring apparatus (Fukuda IR28; weight $27 \mathrm{~g}, 43.5$ (W) $\times 31.5$ (H) $\times 27$ (D) $\mathrm{mm}$ ): a foam-filled flexible cap- sule which was strapped to the right upper abdominal wall and connected to a capacitance manometer (Fig. 1). The sensor was inconspicuously located on the abdomen. Analysis was carried out at 110 times realtime using an electrocardioscanner (SCM $280-4$ ), and the $24 \mathrm{~h}$ compressive wave of the electrocardiogram and respiratory movement were printed out. Apnea was defined as a cessation of abdominal wall movement lasting at least $10 \mathrm{sec}$. SRBD was diagnosed if at least 30 apneic episodes were observed during sleep (Fig. 2)6 Central-type and mixed-type sleep apnea patients were both included in our study. All of the patients were allowed to perform their normal daily activities and to take their usual drugs. Sleeping time was based on daily diaries. Ventricular arrhythmias and silent myocardial ischemia (SMI) were analyzed in each patient and examined in relation to the occurrence of SRBD. SMI was defined as horizontal or downsloping ST-segment depression of at least $0.1 \mathrm{mV}$ that lasted $60 \mathrm{sec}$ or longer.

\section{Follow-up}

Patients were followed up for a mean period of $18.4 \pm 7.6$ months (range $3.2-31.7$ months) after the first recording. The car- 


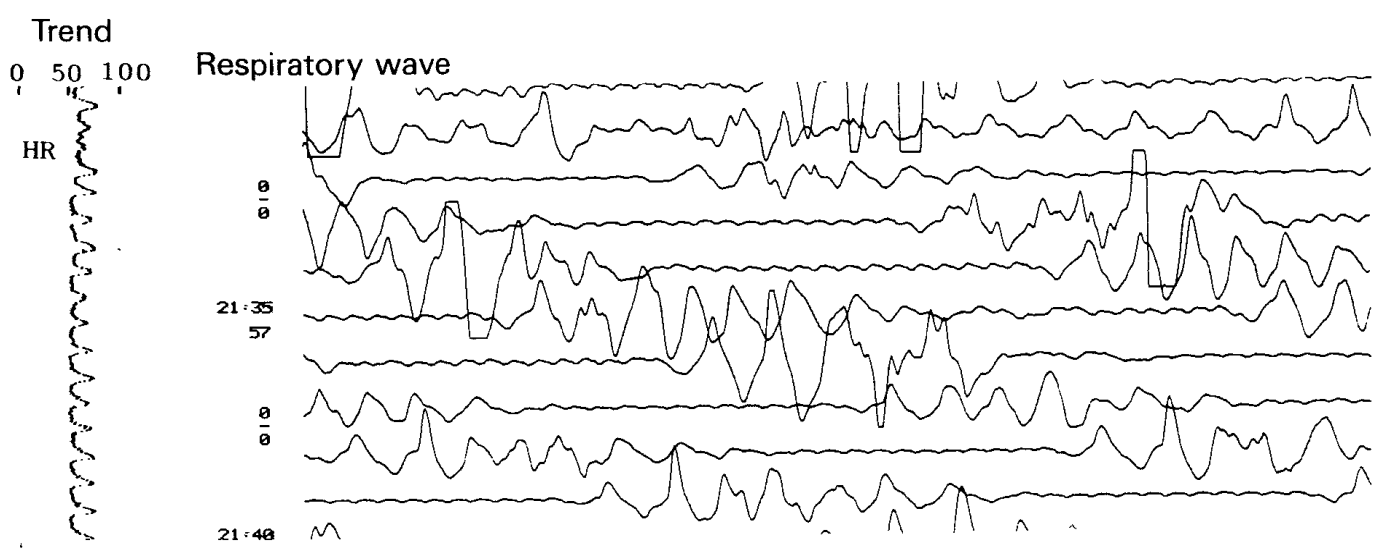

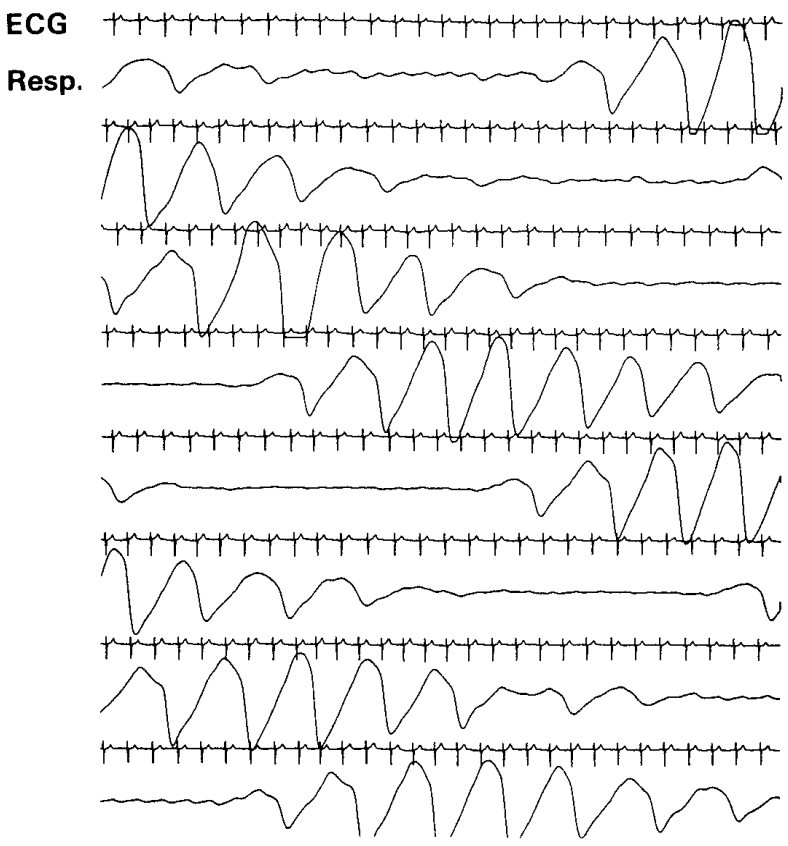

diac events evaluated during follow-up included occurrence of sudden death, myocardial infarction and ventricular tachycardia. Ventricular tachycardia was defined as 3 or more consecutive beats of ventricular origin observed by $24 \mathrm{~h}$ ambulatory electrocardiogram during follow-up.

\section{Statistical Analysis}

The unpaired t-test or $\chi^{2}$ analysis was used to assess differences in data, and $\mathrm{p}<0.05$ was regarded as statistically significant for all analyses. All data are presented as the mean value $\pm \mathrm{SD}$.

\section{RESULTS}

\section{Baseline Clinical Characteristics}

SRBD was detected in 9 of 85 patients $(11 \%)$. The baseline clinical characteristics
Fig.2. Trend of heart rate and respiratory wave in apnea

Cyclical variation of heart rate was observed in the heart rate trendgram.

in patients with SRBD (SRBD group) and patients without SRBD (non-SRBD group) are shown in Table I. There were no significant differences between the two groups with respect to age, sex, body mass index [body weight $(\mathrm{Kg}) /$ height $\left.(\mathrm{m})^{2}\right]$, hypertension, use of beta-blocking drugs, active smoking, history of diabetes, severity of coronary artery stenosis, or prior myocardial infarction. However, more patients had low EF $(\mathrm{EF}<50 \%)$ in the SRBD than in the nonSRBD group $(\mathrm{p}<0.01)$.

Ventricular Arrhythmia and Silent Myocardial Ischemia

The number of ventricular premature contractions (VPC) observed during the study was $403 \pm 475$ in the SRBD group and $176 \pm 650$ in the non-SRBD group (Table II). Of these, complex VPC (Lown 3 or more) were observed in 2 patients $(22 \%)$ in the SRBD group and in 6 patients $(8 \%)$ in the non-SRBD group. There was no significant difference between the two groups. SMI was observed in 4 patients $(5 \%)$ in the nonSRBD group and none in the SRBD group. Again, there was no statistically significant difference between the two groups. The results were similar when the relations with the occurrence of SRBD were examined for only sleeping time.

\section{Clinical Outcomes}

Seventy-seven of the 85 patients (91\%) were followed throughout the duration of the study. Eight patients (all in the non- 
TABLE I BASIC CHARACTERISTICS

\begin{tabular}{lccc}
\hline \hline Variables & $S R B D$ & No SRBD & $P$ \\
\hline Number of patients & 9 & 76 & \\
Age (years) & $57 \pm 9$ & $60 \pm 11$ & $N S$ \\
Malelfemale ratio (\%) & $9 / 0(100)$ & $72 / 4(95)$ & $N S$ \\
Body mass index (kg/m $\left.{ }^{2}\right)$ & $24 \pm 3$ & $24 \pm 2$ & $N S$ \\
Hypertension (\%) & $3(33)$ & $30(40)$ & $N S$ \\
Beta-blocking agent (\%) & $2(22)$ & $12(16)$ & $N S$ \\
Smoking (\%) & $5(56)$ & $37(49)$ & $N S$ \\
Diabetes mellitus (\%) & $19(25)$ & $N S$ \\
Multivessel disease (\%) & $20(32)$ & $N S$ \\
Prior myocardial infarction (\%) & $5(44)$ & $32(42)$ & $N S$ \\
Ejection fraction $<50 \%(\%)$ & $6(63)$ & $5(7)$ & 0.01 \\
\hline
\end{tabular}

SRBD: Sleep-related breathing disorder

TABLE II VENTRICULAR ARRHYTHMIA AND SILENT MYOCARDIAL ISCHEMIA

\begin{tabular}{lccc}
\hline \hline Variable & SRBD & No SRBD & $P$ \\
\hline Number of patients (cases) & 9 & 76 & \\
Number of VPC ( /day) & $403 \pm 475$ & $176 \pm 650$ & $N S$ \\
Complex VPC (cases (\%)) & $2(22)$ & $6(8)$ & $N S$ \\
SMI (cases (\%)) & $0(0)$ & $4(5)$ & $N S$ \\
\hline
\end{tabular}

VPC: ventricular premature contraction, SMI: silent myocardial ischemia

TABLE III CARDIAC EVENTS

\begin{tabular}{lccc}
\hline \hline Variable & SRBD & No SRBD & $P$ \\
\hline Number of patients & 9 & 68 & \\
Follow up (month) & $17.3 \pm 7.3$ & $17.4 \pm 7.7$ & $N S$ \\
Cardiac event & $4(44)$ & $4(6)$ & 0.001 \\
Total (\%) & $1(11)$ & $1(1)$ & $N S$ \\
Sudden death (\%) & $3(33)$ & $3(4)$ & 0.002 \\
VT (\%) & & & \\
\hline
\end{tabular}

$V T:$ ventricular tachycardia

SRBD group) could not be followed because they moved abroad or elsewhere in Japan. During follow-up, sudden death occurred in 2 patients, and ventricular tachycardia was recorded in 6 patients (Table III). New onset myocardial infarction did not occur during the study. Four of $9(44 \%)$ patients in the SRBD group had cardiac events, compared with 4 of $79(6 \%)$ patients in the non-SRBD group $(p<0.001)$. Neither the follow-up period nor the number of $24 \mathrm{~h}$ ambulatory electrocardiogram recordings during follow-up (SRBD group: $2.6 \pm 2.2$ times, non-SRBD group: $2.3 \pm 1.5$ times) was significantly different between the two groups.

\section{DISCUSSION}

Olazabal et al, Otsuka et $\mathrm{al}^{5}$ and Hung et $\mathrm{al}^{7}$ reported that sleep apnea was prone to occur in patients with coronary artery disease. However, there has been no study of the incidence of SRBD in patients with coronary artery disease. In our study, SRBD was found in $11 \%$ of the 85 coronary artery patients, although the actual incidence of SRBD in association with coronary artery disease should be higher because obstructive-type SAS and some mixed-type SAS were not included in our study.

There has been no previous report of the prognosis of coronary artery patients with SRBD. Our study showed that the SRBD 
group was prone to cardiac events, such as ventricular tachycardia or sudden death, and their EF was much lower than that in the non-SRBD group. There are at least two possible explanations for the poor prognosis in SRBD patients. First, SRBD itself may have aggravated the prognosis. It is well known that hypoxia and various hemodynamic disturbances, such as blood pressure elevation, heart rate change and arrhythmia, occur during apnea episodes $1-5,8$ This incessant cardiac stress every night may worsen the prognosis of coronary artery patients with SRBD. This is noteworthy because SRBD can be treated 9,10 and such treatment might decrease the occurrence of cardiac events 11 The second possibility is that a lower cardiac function may have provoked SRBD, and the poor prognosis in the SRBD group may have been due to their lower cardiac function. Yasuma et $a^{12}$ reported that a delayed circulation time was one of the predisposing factors for the occurrence of SRBD in stable chronic heart failure. This suggests the possibility that a delayed circulation time caused by poor cardiac function induced SRBD, and this poor cardiac function enhanced the risk for cardiac events. ${ }^{13}$ We could not determine if SRBD was an independent risk factor for future cardiac events. However, SRBD should be considered as a warning sign for future cardiac events in coronary artery patients.

Monitoring of SRBD with AERMS (Fukuda SM28/IR28) was convenient and inexpensive, since respiratory waves could be easily recorded by simply attaching a respiratory monitoring apparatus to a conventional ambulatory ECG recorder. Furthermore, ordinary outpatient studies could be performed on a conventional ambulatory ECG basis, since the sensor was inconspicuously located on the right upper abdomen. Due to its clinical usefulness, convenience and low cost, AERMS was considered to be a valuable tool for monitoring SRBD patients. However, one limitation is that only centraltype and some mixed type sleep apnea pa- tients could be detected by AERMS.

Further controlled trials will be needed before the clinical significance of AERMS is thoroughly clarified.

\section{REFERENCES}

1. SHEPARD JW: Cardiorespiratory changes in obstructive sleep apnea. In: Kryger $\mathrm{MH}$, Roth $\mathrm{T}$, Dement WC eds. Principles and Practice of Sleep Medicine, Philadelphia, Saunders 1989; 537-551

2. GUILLEMINAULT C, CONNOLLY SJ, WINKLE RA: Cardiac arrhythmia and conduction disturbances during sleep in 400 patients with sleep apnea syndrome. Am J Cardiol 1983; 52: 490-494

3. SHEPARD JW, GARRISON MW, GRITHER DA, DOLAN GF: Relationship of ventricular ectopy to oxyhemoglobin desaturation in patients with obstructive sleep apnea. Chest 1985; 88: $335-340$

4. OLAZABAL JRD, MILLER MJ, COOK WR, MITHOEFER JC: Disordered breathing and hypoxia during sleep in coronary artery disease. Chest 1982; 82: 548-552

5. OTSUKA $\mathrm{K}$, SADAKANE $\mathrm{N}$, OZAWA $\mathrm{T}$ : Arrhythmogenic properties of disordered breathing during sleep in patients with cardiovascular disorders. Clin Cardiol 1987; 10: 771-782

6. GUILLEMINAULT C, TILKIAN A, DEMENT WC: The sleep apnea syndromes. Annu Rev Med 1976; 27: 465-484

7. HUNG J, WHITFORD EG, PARSONS RW, HILLMAN DR: Association of sleep apnoea with myocardial infarction in men. Lancet 1990; 336: $261-264$

8. BLOCK AJ: Dangerous sleep: Oxygen therapy for nocturnal hypoxemia. $N$ Engl J Med 1982; 306: $166-167$

9. KRYGER MH: Management of obstructive sleep apnea. In: Kryger MH, Roth $\mathrm{T}$, Dement WC eds. Principles and Practice of Sleep Medicine. Philadelphia, Saunders, 1989; 584-590

10. WHITE DP: Central sleep apnea. In: Kryger MH, Roth T, Dement WC eds. Principles and Practice of Sleep Medicine. Philadelphia, Saunders, 1989; 513-524

11. PARISH JM, SHEPARD JW: Cardiovascular effects of sleep disorders. Chest 1990; 97: $1220-1226$

12. YASUMA F, NOMURA H, HAYASHI H, OKADA T, TSUZUKI M: Breathing abnormalities during sleep in patients with chronic heart failure. Jpn Circ J 1989; 53: $1506-1510$

13. MATTIONI TA: Long-term prognosis after myocardial infarction. Postgrad Med 1992; 92: $107-114$ 